\title{
Author Index Vol. 35, 1990
}

Aitkin, L.M. 325 BagnoH,P. 31,40 Bartheld, CS. von 1 Bass,A.H. 350 g|San,J. 350 Behrend, K. 227 Bell,K. 156 Bleckmann,H. 240 Brands^ffir, R, 195 Browman, H.I. 85 Bullock, T.H. 278,291,313 Buttery, R.G. 156 Bgasini, G. 31 Cheverud, J.M. 368 Clairambault, P. 212 Comfort, N.C. 350 Derby, CD. 129 Donicht, M. 227 Douglas, R.H. 98 Dub6,L. 212 Evans, Bfi. 85 Falk,D. 368 
Finger, T.E. 268 E^Sis,S. 176 Fontanesi, G. 31,40 K.CA. 302**; KgSrdot, M.-N. 129 Gordon, W.C. 85 GuerraSeTi^iM.J. 16 Hagedorn,M. 268 Haight,J.R. 156 Helmkamp, R.C. 368 Hildebolt, C. 368 Hopkins, CD. 350 Hornby, P.J. 49 Jonker, A.J. 65 ||Sg|Kl,K. 195 Ha,D. 146 Labandeira-GarclaWA- 16 Labandeira-Garcfa, J.L. 16 Lee,L.T. 278,291,313 Malacarne, G. 212 Manteuffel, G. 176 EMJose. P.A. 98 
Moore, A.J. 368 EtuSz,H. 240 Murakami, D.M. 302 O'Brien, W.J. 85 0'Shea,T.J. 185 Piekut,D.T. 49 Plogmann, D. 146 Eoiciatti, V. 31,40 Raffaelli.A. 40 Reep, R.L. 185 Rowe, M.H. 253 Sanderson, KJ. 325,339 Sevensma, JKT; 65 K§S,S.R. 107 Smeets, VVJ.A.J. 65 Teyke,T. 23 Vannier,M. 368 Walker, R.F. 98 KjjS|r,W.L. 339 WomblelS. 268 